\title{
The Research on Wireless Sensor Network for Mechanical Vibration Monitoring
}

\author{
Xin-min $\mathrm{CAO}^{1, \text { a }}$ \\ ${ }^{1}$ Guangxi Technical College of Machinery and electricty, Nanning 530007, China \\ axinmincao@126.com
}

Keywords: Vibration Monitoring, Wireless Sensor Network, Mechanical Vibration

\begin{abstract}
The mechanical equipment especially large key equipment reliability, availability, maintainability, and safety is related to enterprise's production efficiency and people's life and property security, wireless sensor network (WSN) is used to build the mechanical vibration monitoring system can make up for the inadequacy of traditional cable monitoring system. Wireless sensor network technology is applied in mechanical vibration monitoring and efficient to solve the high frequency vibration signal to collect real-time, high speed data acquisition, data storage, and reliable data transmission and so on. Most of the existing general wireless sensor network node hardware performance is not high, poor operation and processing capacity, is difficult to meet the monitoring requirements of mechanical vibration, mechanical vibration monitoring for wireless sensor networks, this paper carried out research.
\end{abstract}

\section{Introduction}

Wireless sensor network (WSN) is developed in recent years, the emerging field of study, it will be of perception, communication, computing nodes densely deployed in the monitored area, access to the collection and handling of the regional environmental parameters to monitor or to monitor the working state of the object [1]. Wireless sensor networks in military, environment monitoring, health care, intelligent transportation, and intelligent household and other fields has a very wide range of applications, because in the field of mechanical vibration monitoring high speed, high precision synchronous pick face machinery vibration signal acquisition and reliable data storage and transmission, on a chip processing capacity, energy supply, and the absence of a set of suitable for mechanical equipment condition monitoring system, make its application is blocked, once these problems are solved, the wireless sensor network in the field of mechanical vibration monitoring will have great potential applications [2].

Wireless sensor network is composed of a large number of nodes deployed in the monitoring area, these nodes by wireless communication form a self-organization monitoring network system, able to real-time monitoring, collaboration awareness and acquisition of various environmental or monitoring object information, and through the embedded system in information processing, finally through the self-organized wireless network transmission to the user terminal, which plays a role of support technology of Internet of things. Mechanical vibration monitoring based on wireless sensor network (WSN) is applied to face the difficulties of study, the design of wireless sensor network (WSN), the better for wireless sensor network is applied in mechanical vibration monitoring is of great importance.

\section{Summary of wireless sensor network}

As the underlying network is an important part of the Internet, the U.S. military initially proposed a wireless sensor networks (Wireless Sensor Network) the research of theory and application. Wireless sensor network (WSN) consists of a large number of sensor nodes deployed in the monitoring region of communicating with each other, forming a multi hop ad hoc network system [3]. Wireless communication, sensor technology, embedded application and microelectronic technology, wireless sensor network is increasingly mature can at any time, any place, and any environmental condition for the people the information they need, to lay the foundation for the 
development of the internet. Because the network deployment, self-organization, rapid, high fault tolerance and strong concealment, and so on, so it is very suitable for the battlefield target positioning, planetary exploration, physiological data acquisition and monitoring, and many other fields. The network architecture is shown in figure 1.

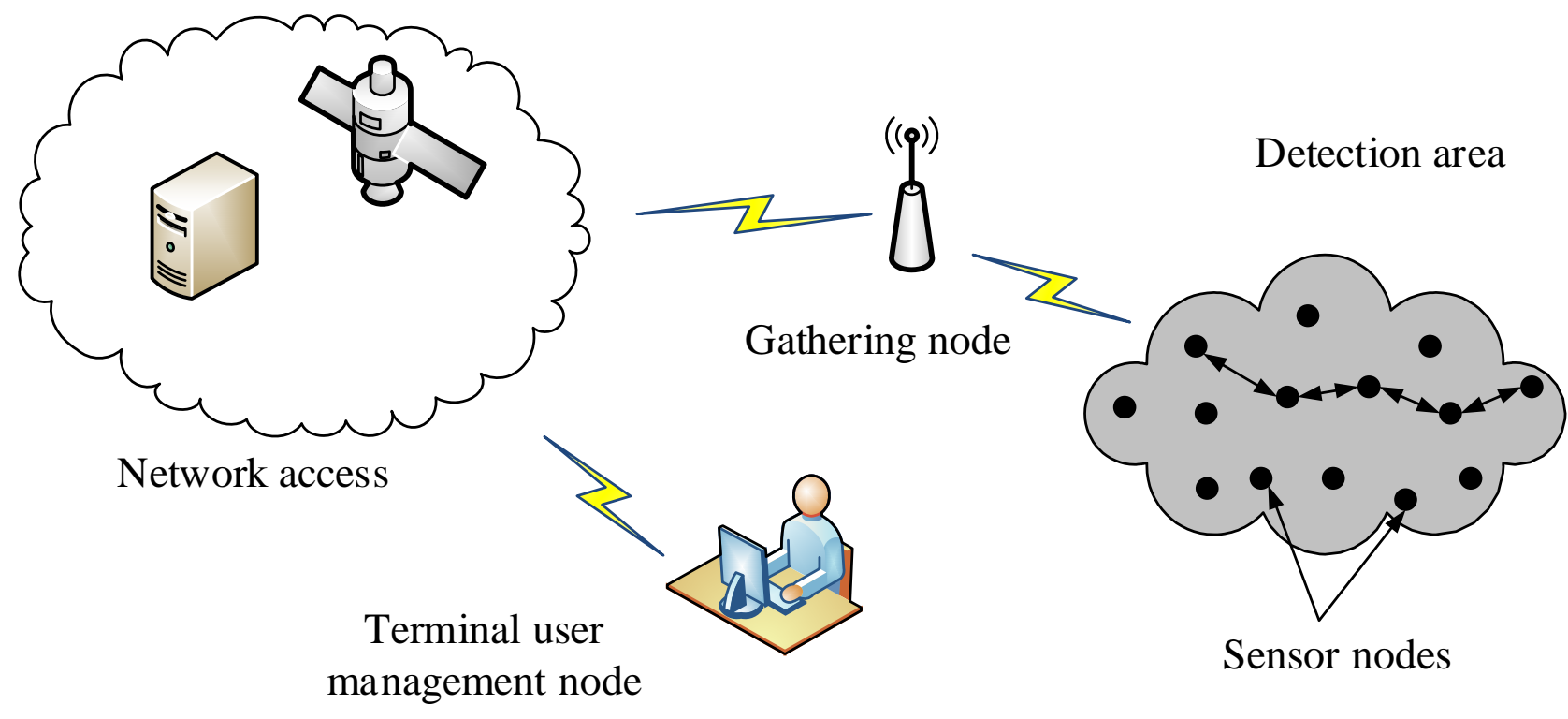

Figure 1.Architecture of wireless sensor network system

Sensor deployment is random distribution of a large number of forms or manually placed in the monitoring area, the construction of self-organized [4] through network. The sensor node through the network monitoring field data in the transmission of multi hop routing node collection nodes, multi node data may be fusion and compression, in the transport process finally, through satellite Internet or wireless access server terminal management node. Users can configure management, network management node releases and task feedback safety control operation.

\section{Monitoring system node hardware platform design}

To pick up to solve mechanical vibration signal of high precision and high sampling frequency, as well as the high speed and high precision acquisition brings a large amount of data and issues such as energy consumption, the node can decide the performance of wireless sensor network applications in the field of mechanical vibration monitoring [5]. In this paper, design of mechanical vibration sensor network node overall scheme and hardware architecture as shown in figure 2, the node has high computing performance, lower power consumption, and mass data storage capacity. Node by the data acquisition module, data storage module, wireless communication module, power module and processor module and so on six module units, adopting modular, the design idea of replaceable combination, convenient user according to the specific requirements and cost requirements of monitoring objects chooses appropriate hardware combination plan.

Wireless sensor network designed in this paper the mechanical vibration node hardware platform is based on A dual processor architecture design scheme of the Cortex M3 kernel 32-bit ARM processor and low power wireless rf chip CC2430 as the core, with 16 bits A/D converter, MEMS acceleration sensor, mass storage, wireless rf module and power module to realize the collection of mechanical vibration signals. In mechanical vibration signal of the wireless sensor network node hardware design, is based on the last generation of node power supply and signal conditioning module made improvements, and four layers PCB design scheme is adopted to make the node on the electromagnetic compatibility and volume had the very big improvement, is laid a good foundation for further study of the thesis. 


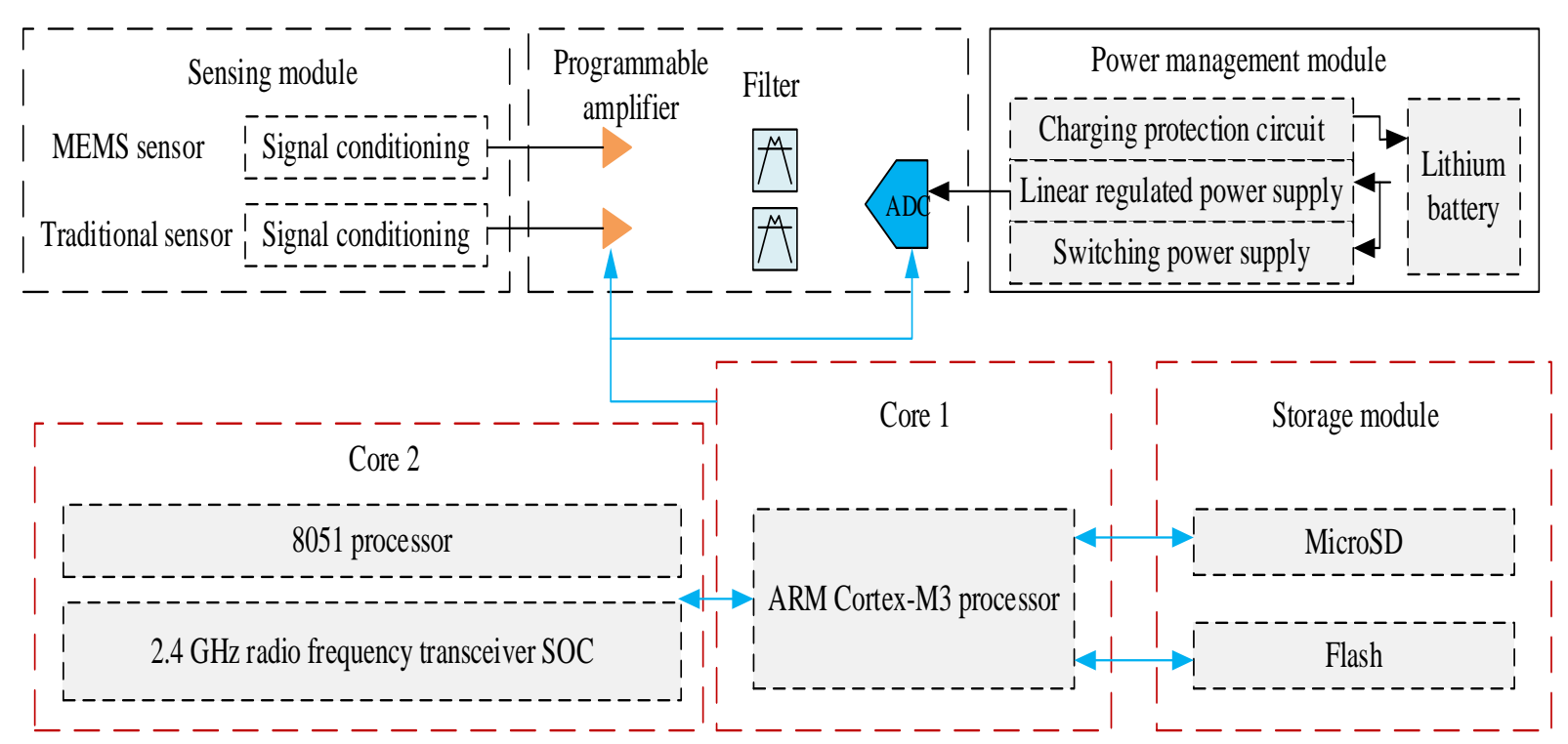

Figure 2.The overall block diagram of the hardware scheme

\section{The overall architecture of monitoring system software design}

Node software design were introduced in this paper, involving the transplanting of embedded operating system, a child module driver development, and application to write, to realize the collection of vibration signal, and the acquisition of vibration signal on a chip processor design, due to the node energy and computation ability is limited, the software design must always consider the design features of high efficiency low power consumption. Monitoring system software design of overall architecture as shown in figure 3, division two processor module node cooperation, including 1 processor is responsible for data acquisition, storage, handling, and file system management, processor is responsible for running the wireless communication protocol, 2 complete node self-organization, such as time synchronization and data sending and receiving task.

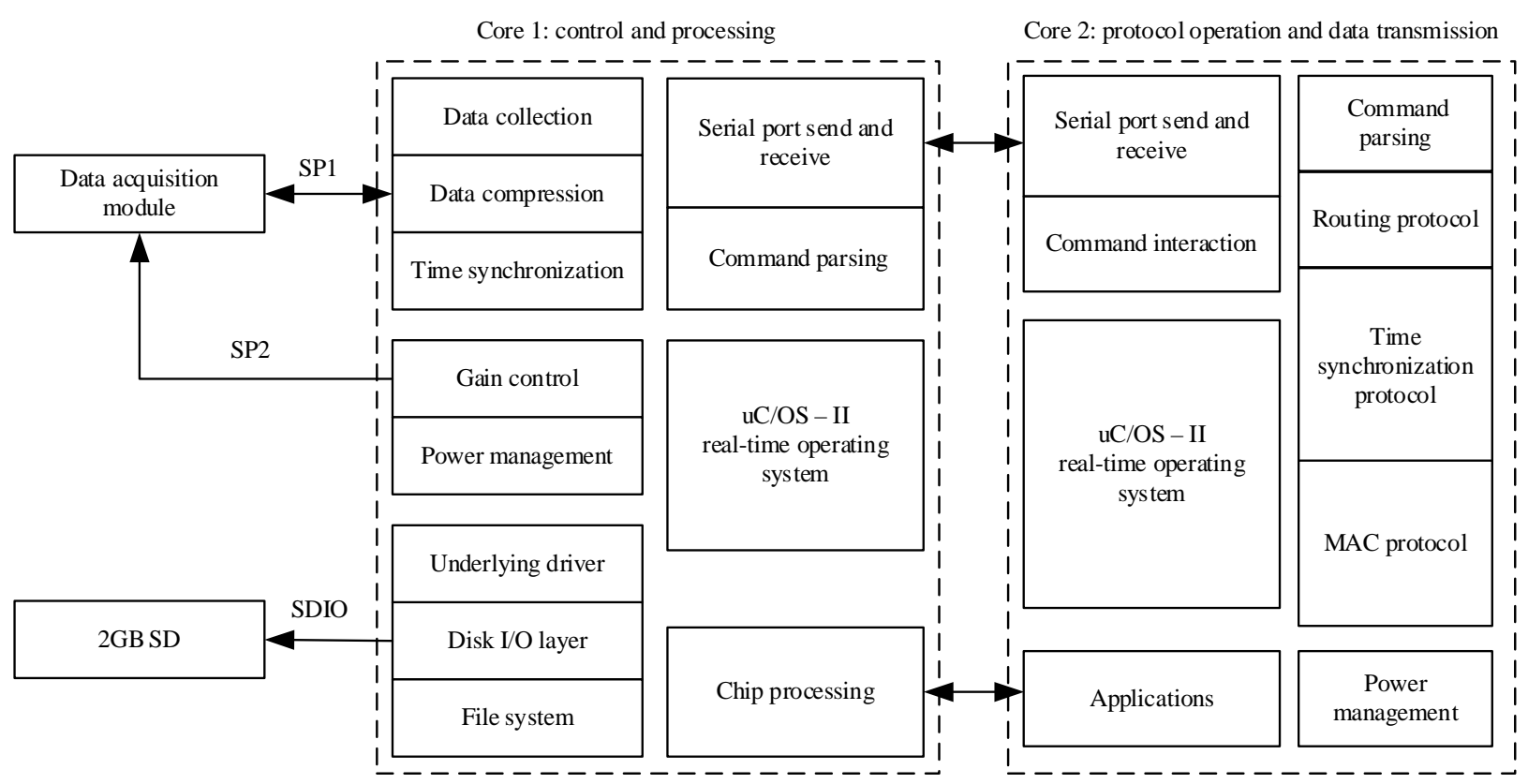

Figure 3. The overall block diagram of the software scheme

In order to give full play to the processor performance, two processors were implanted with uC/OS - II real-time operating system, operating system like a bridge of communication applications and the underlying hardware, the underlying hardware driver operating the system to provide the API function, makes the application software has nothing to do with the underlying 
hardware and the application of writing from the hardware, improve the portability and readability of the program, thereby lowering the cost of system development and shorten the development cycle. Processor 1 in the underlying hardware drive mainly includes data acquisition interface function, file read/write interface function, and low power consumption mode interface functions, applications, including common signal characteristic analysis method and characteristic parameter extraction, the processor in the 2 wireless communication module developed by other team members. Dual processor via a serial port to commands and data exchange between dual processor do their job and collaboration, enhance the overall performance of the node and can reduce the coupling between the hardware and software modules.

\section{Conclusion}

With the rapid development of industrial production and science and technology, on the degree of precision mechanical equipment, complexity, and demand more and more high degree of automation, equipment reliability, maintainability and safety problem increasingly prominent, more and more people study has been carried out on mechanical equipment vibration monitoring technology, has introduced a variety of mechanical vibration monitoring system at home and abroad, to a certain extent to ensure the security and stability of the mechanical equipment operation. Mechanical condition monitoring based on wireless sensor network (WSN), implements the multi-node ad-hoc network and data transmission, formed a complete wireless sensor network (WSN) machinery and equipment condition monitoring system, and the monitoring system has carried on the experiment and application.

\section{References}

[1] B. Lu, V.C. Gungor: Industrial Electronics, IEEE Transactions on, Vol.56 (2009) No.11, p. 4651.

[2] F. Stajano, N. Hoult, I. Wassell: Ad Hoc Networks, Vol.8(2010) No.8, p. 872.

[3] P. Li, Y. Wen, P. Liu: Sensors and actuators A: Physical, Vol.157 (2010) No.1, p. 100.

[4] B. Aygün, V.C. Gungor: Sensor Review, Vol.31 (2011) No.3, p. 261.

[5] E. Gilbert, B. Kaliaperumal, E.B. Rajsingh: International Journal of information and electronics engineering, Vol.2 (2012) No.5, p. 702. 